\title{
Making Space-Time Crystals Using Magnons
}

\author{
Electron spin waves condense to form an exotic new state that repeats in \\ both space and time.
}

By Sophia Chen

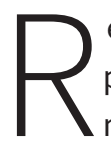
esearchers in the field of spintronics aim to exploit the properties of materials' electron spins to develop new, more energy-efficient information technologies. Such schemes often involve encoding and transmitting data via quasiparticles called magnons-collective excitations of electron spins. Now, Joachim Gräfe, of the Max Planck Institute for Intelligent Systems, Germany, and colleagues have used magnons to form a new state of matter called a space-time crystal, and they have studied how this state interacts with other magnons [1].

Just as a conventional crystal is defined by a pattern that repeats in space, a space-time crystal has a varying structure that also repeats in time. Such structures have only recently been realized experimentally (see Viewpoint: How to Create a Time Crystal). Gräfe and colleagues created their space-time crystal by applying a radio-frequency field to a micrometer-scale, room-temperature strip of nickel-iron alloy. The field excited magnons that formed a dynamic spatial pattern, which the researchers liken to an arrangement of balls

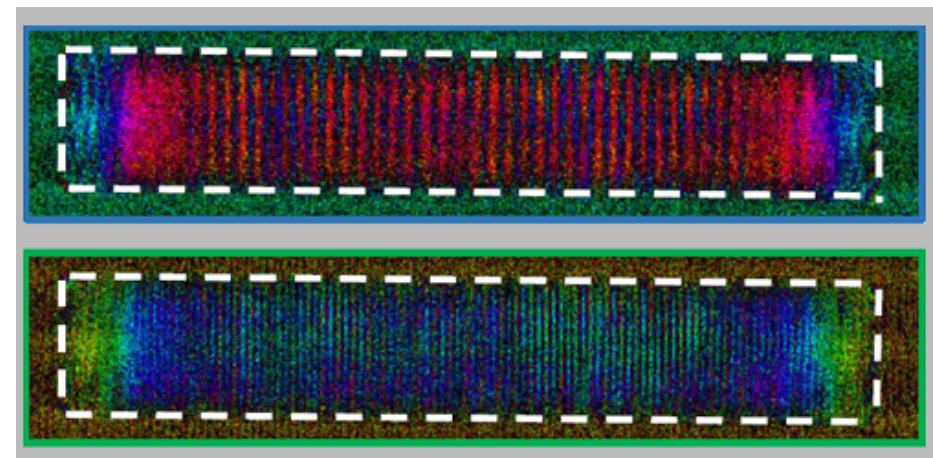

Credit: N. Träger et al. [1] on a billiard table-if billiard balls returned repeatedly to their initial collective state after dispersing.

After imaging the space-time crystal using x-ray microscopy, the researchers directed other magnons toward it. They found that these magnons were scattered similarly to how they would be from a conventional crystal. This scattering process produced ultrashort magnons whose precise wavelengths could be tuned by changing the parameters of the radio-frequency field. The researchers say that the ability to easily reconfigure the space-time crystal in this way, coupled with its room-temperature operation, make the device a suitable platform for magnon-based information technology.

Sophia Chen is a freelance science writer based in Columbus, Ohio.

\section{REFERENCES}

1. N. Träger et al., "Real-space observation of magnon interaction with driven space-time crystals," Phys. Rev. Lett. 126, 057201 (2021). 